\title{
Structure and mechanism of the iron-sulfur flavoprotein phthalate dioxygenase reductase
}

\author{
GEORGE T. GASSNER, MARTHA L. LUDWIG, DOMENICO L. GATTI, CARL C. CORRELL, ${ }^{1}$ AND \\ DAVID P. BALLOU ${ }^{2}$ \\ Department of Biological Chemistry and Biophysics Research Division, University of Michigan, Ann Arbor, \\ Michigan 48109, USA
}

\begin{abstract}
The oxygenation of unactivated aromatic compounds is a common event in soil bacteria and is very important for the environmental breakdown of such compounds. The phthalate diooxygenase system from Pseudomonas cepacia provides a typical example of the reactions that occur in such metabolic pathways. Here phthalate is converted to its cis-dihydrodiol, with the incorporation of molecular oxygen atoms and using $\mathrm{NADH}$ as a source of reducing equivalents. The phthalate diooxygenase system is composed of two enzymes: a terminal dioxygenase that contains a Rieske iron-sulfur center and a ferrous iron center, and an iron-sulfur flavoprotein reductase that is responsible for transfer of reducing equivalents from NADH to the dioxygenase. In this article the authors present a detailed analysis of the events occurring when NADH reacts with the reductase, tracing the sequence of hydride and electron transfer reactions among the two redox centers and relating these steps to known crystal structure forms of the enzyme.
\end{abstract}

ABSTRACT Transfer of electrons between pyridine nucleotides (obligatory two-electron carriers) and hemes or [2Fe-2S] centers (obligatory one-electron carriers) is an essential step mediated by flavins in respiration, photosynthesis, and many oxygenase systems. Phthalate dioxygenase reductase (PDR), a soluble iron-sulfur flavoprotein from Pseudomonas cepacia, is a convenient model for the study of this type of electron transfer. PDR is folded into three domains; the $\mathrm{NH}_{2}$-terminal FMN binding and central $\mathrm{NAD}(\mathrm{H})$ binding domains are closely related to ferredoxin-NADP ${ }^{+}$reductase (FNR). The COOH-terminal [2Fe-2S] domain is similar to plant ferredoxins, and can be removed by proteolysis without significantly altering the reactivity of the FNR-like domains. Kinetic studies have identified sequential steps in the reaction of PDR with NADH that involve pyridine nucleotide binding, hydride transfer to FMN, and intramolecular electron transfer from the reduced flavin to the [2Fe-2S] cluster. Crystal structures of reduced and liganded PDR correspond to some of the intermediates formed during reduction by NADH. Small structural changes that are observed in the vicinity of the cofactors upon reduction or $\operatorname{NAD}(H)$ binding may provide part of the reorganization energy or contribute to the gating mechanism that controls intramolecular electron transfer.-Gassner, G. T., Ludwig, M. L., Gatti, D. L., Correll, C. C., Ballou, D. P. Structure and mechanism of the iron-sulfur flavoprotein phthalate dioxygenase reductase. FASEB J. 9, $1411-1418$ (1995)

Key Words: iron-sulfur protein $\cdot$ electron transport $\cdot$ pyridine nucleotide

PYRIDINE NUCLEOTIDES PROVIDE A SOLUBLE POOL of reducing equivalents for cellular metabolic activities. Electron transfer chains involved in respiration, photosynthesis, and oxygenation of unactivated aromatic compounds are all able to exchange electrons with the pyridine nucleotide pool via the transfer of hydrides between $\operatorname{NAD}(\mathrm{H})$ or $\mathrm{NADP}(\mathrm{H})$ and flavin containing enzymes. The phthalate dioxygenase system from Pseudomonas cepacia is a representative multienzyme complex that catalyzes such an exchange. In this system phthalate is converted to its cis-dihydrodiol, utilizing molecular oxygen and reducing equivalents from NADH. Similar cis-dihydroxylations of aromatic compounds are catalyzed by related dioxygenase systems in other bacteria.

Each of the known dioxygenase systems uses an FAD- or FMN-containing reductase, an electron transfer array of $[2 \mathrm{Fe}-2 \mathrm{~S}]^{3}$ centers, and a terminal dioxygenase that requires ferrous iron $(1,2)$. In addition to the ferrous iron, which is the site of oxygenation, each oxygenase compo-

\footnotetext{
'Current address: Department of Biophysics and Biochemistry, Yale University, New Haven, CT 06511, USA.

${ }^{2}$ To whom correspondence should be addressed, at: Department of Biological Chemistry, University of Michigan, Ann Arbor, MI 48109 0606, USA.

${ }^{3}$ Abbreviations: [2Fe-2S], two iron, two sulfur center; PDR, phthalate dioxygenase reductase; FNR, ferredoxin $\mathrm{NADP}^{+}$-reductase; $\mathrm{PDR}(-\mathrm{FeS})$, $\mathrm{PDR}$ that lacks the $10 \mathrm{kDa}$ [2Fe-2S] domain; $\mathrm{SQ}$, flavin semiquinone and reduced [2Fe-2S] form of PDR; $\mathrm{PDR}_{2 \mathrm{e} \text {.; }} \mathrm{PDR}_{3 \mathrm{e} \text {; }}$ two- and three-electron reduced forms of PDR; MC-1, MC-2, CT-1, CT*. Michaelis and chargetransfer complexes defined in Scheme 1 and in the text; FMN ${ }_{\mathrm{ox}}, \mathrm{FMN}_{\mathrm{sq}}$, FMN $N_{\text {liq }}$ oxidized, semiquinone, and reduced forms of the FMN bound to PDR phthalate; PDO, dioxygenase.
} 
TABLE 1. Bacterial dioxygenase systems ${ }^{a}$

\begin{tabular}{|c|c|c|c|}
\hline Type & Reductase & $\begin{array}{l}\text { Intermediate } \\
\text { Electron Transfer }\end{array}$ & Oxygenasae \\
\hline I & FMN/Cys4 $[2 \mathrm{Fe}-2 \mathrm{~S}]$ & None & $\begin{array}{c}\mathrm{Cys}_{2} \mathrm{His}_{2}[2 \mathrm{Fe}-2 \mathrm{~S}] \\
\mathrm{Fe}^{2+}\end{array}$ \\
\hline $\mathbf{I}_{\boldsymbol{A}}$ & FAD/Cys4 $[2 \mathrm{Fe}-2 \mathrm{~S}]$ & None & $\begin{array}{c}\mathrm{Cys}_{2} \mathrm{His}_{2}[2 \mathrm{Fe}-2 \mathrm{~S} \\
\mathrm{Fe}^{2+}\end{array}$ \\
\hline II & FAD & $\mathrm{Cys}_{4}[2 \mathrm{Fe}-2 \mathrm{~S}]$ & $\begin{array}{c}\mathrm{Cys}_{2} \mathrm{His}_{2}[2 \mathrm{Fe}-2 \mathrm{~S}] \\
\mathrm{Fe}^{2+}\end{array}$ \\
\hline II $_{\mathbf{A}}$ & FAD & $\mathrm{Cys}_{2} \mathrm{His}_{2}[2 \mathrm{Fe}-2 \mathrm{~S}]$ & $\begin{array}{c}\mathrm{Cys}_{2} \mathrm{His}_{2}[2 \mathrm{Fe}-2 \mathrm{~S}] \\
\mathrm{Fe}^{2+}\end{array}$ \\
\hline III & FAD/Cys4 [2Fe-2S] & $\mathrm{Cys}_{2} \mathrm{His}_{2}$ [2Fe-2S] & $\begin{array}{c}\mathrm{Cys}_{2} \mathrm{His}_{2}[2 \mathrm{Fe}-2 \mathrm{~S}] \\
\mathrm{Fe}^{2+}\end{array}$ \\
\hline
\end{tabular}

'Distribution of flavin and metal centers between the reductase, intermediate electron transferase, and oxygenase components of NADH-dependent bacterial dioxygenases.

nent contains a Rieske [2Fe-2S] center $^{4}(3)$. These dioxygenase systems have been classified into three groups based on the number of proteins required in the transfer of electrons from NADH to oxygen and whether there is an iron-sulfur center in the reductase (1) (Table 1). Type I systems, which hydroxylate phthalate, benzoate, $p$-sulfobenzoate, 4-chlorophenylacetate, and 2-halobenzoate (4-8), are composed of only two protein components: a monomeric reductase and a separate multimeric oxygenase. The reductase contains both a flavin and a planttype [2Fe-2S] center. Type II systems, which hydroxylate benzene, toluene, pyrazon, and dibenzofuran (9-12), use a reductase that contains FAD, but no iron-sulfur centers. In these systems, an intermediary Rieske or plant-type ferredoxin transfers electrons from the reductase to the oxygenase. Finally, the reductase components of the type III systems, which hydroxylate naphthalene and $o$-halobenzoate (13-15), are similar to those of type I. Type III systems differ from those of type I due to the presence of an additional small Rieske-type protein that transfers electrons between the reductase and the dioxygenase.

The phthalate dioxygenase system (type I) has two components: phthalate dioxygenase reductase (PDR), a $36 \mathrm{kDa}$ protein with both an FMN and a [2Fe-2S] center; and phthalate dioxygenase (PDO), an oligomeric enzyme with one Rieske center and one mononuclear ferrous iron per $50 \mathrm{kDa}$ monomer (4) (Fig. 1). In this review, we consider the structure of PDR as it relates to the kinetics of the reaction of NADH with the bound FMN and the control of the subsequent intramolecular electron transfer from reduced FMN to the [2Fe-2S] center.

\section{THE STRUCTURE OF PDR, A MEMBER OF THE FNR FAMILY}

The structure of PDR determined by X-ray crystallography reveals a modular arrangement in which the FMN, $\mathrm{NAD}(\mathrm{H})$, and [2Fe-2S] binding sites are associated with

\footnotetext{
${ }^{4}$ The Rieske center has a [2Fe-2S] core, but has two cysteine and two histidine ligands in place of the four cysteine ligands found in plant-type ferredoxins (3).
}

distinct $\mathrm{NH}_{2}$-terminal, central, and $\mathrm{COOH}$-terminal domains (Fig. 2). X-ray analyses of PDR in various redox states and of PDR in complex with NAD, NADH, or pyridine nucleotide analogs have been used to identify the pyridine nucleotide binding site and to characterize structures that may correspond to intermediates in the catalytic cycle of this enzyme (16). As shown in Fig. 2, the $\mathrm{NH}_{2}$-terminal FMN binding domain of PDR is a $\beta$-barrel composed of six strands of antiparallel sheet. FMN is bound to the outside of the barrel in a position adjacent to NADH. The central $N A D(H)$ binding domain is an $\alpha / \beta$ fold, closely resembling the pyridine-nucleotide binding domains of dehydrogenases (17). Comparison of the crystal structures of PDR and of ferredoxin NADP ${ }^{+}$reductase (FNR) indicates that these proteins are close relatives. Although the amino acid sequence identity between PDR and FNR is low $(\sim 15 \%)$, the $\alpha$-carbons of the FMN and NADH binding domains of PDR can be superimposed on the corresponding atoms of the FAD and NADP ${ }^{+}$binding domains of FNR with an rms deviation of only $1.5 \AA$ (18). This structural similarity classifies PDR as a member of the FNR family of flavoprotein reductases (19). Members of the FNR family are distinguished by their novel flavin binding folds, with topologies completely unrelated to the $\alpha / \beta$ flavin binding domains of glutathione reductase and its family of disulfide flavoenzymes $(19,20)$. Sequence homologies identify a number of other proteins, including cytochrome $\mathrm{P}-450$ reductase, sulfite reductase, nitrate reductase, cytochrome $b_{5}$ reductase, and NO synthase, as part of the FNR family $(16,19,21)$. The 3-dimensional structures of nitrate

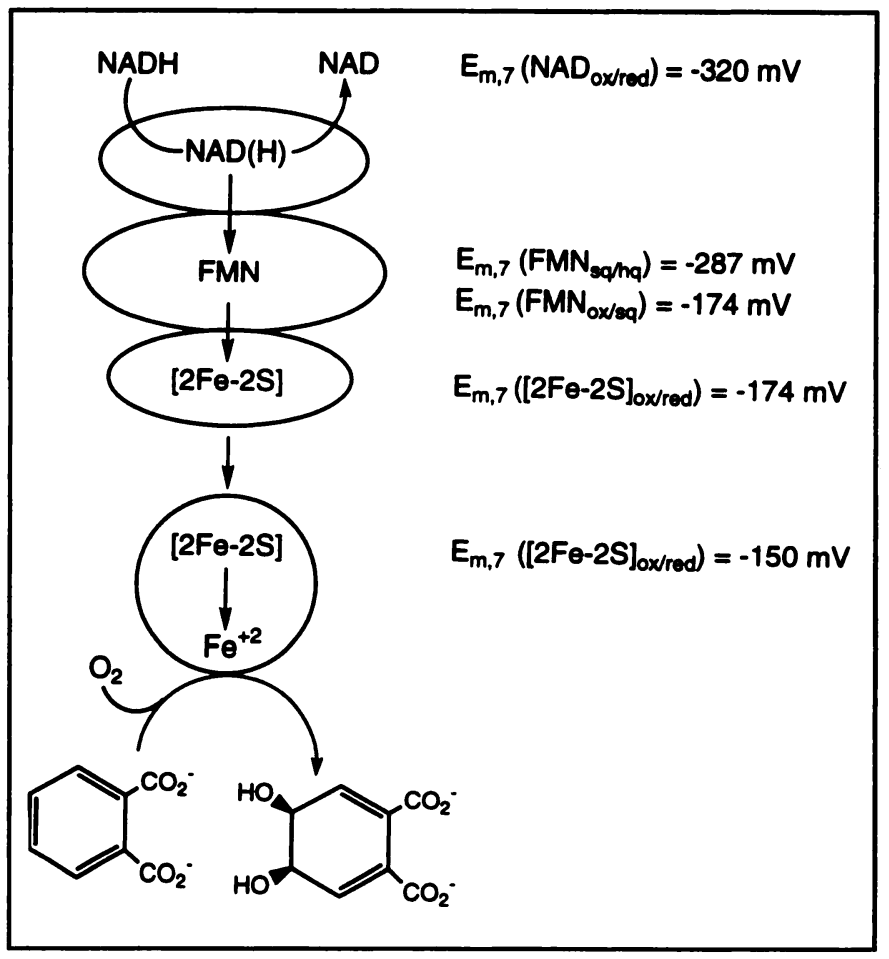

Figure 1. Cartoon of the phthalate dioxygenase system. The three domains of PDR are represented by ellipsoids. A single subunit of PDO is designated by a circle. Inter- and intramolecular electron transfers are represented by arrows. 


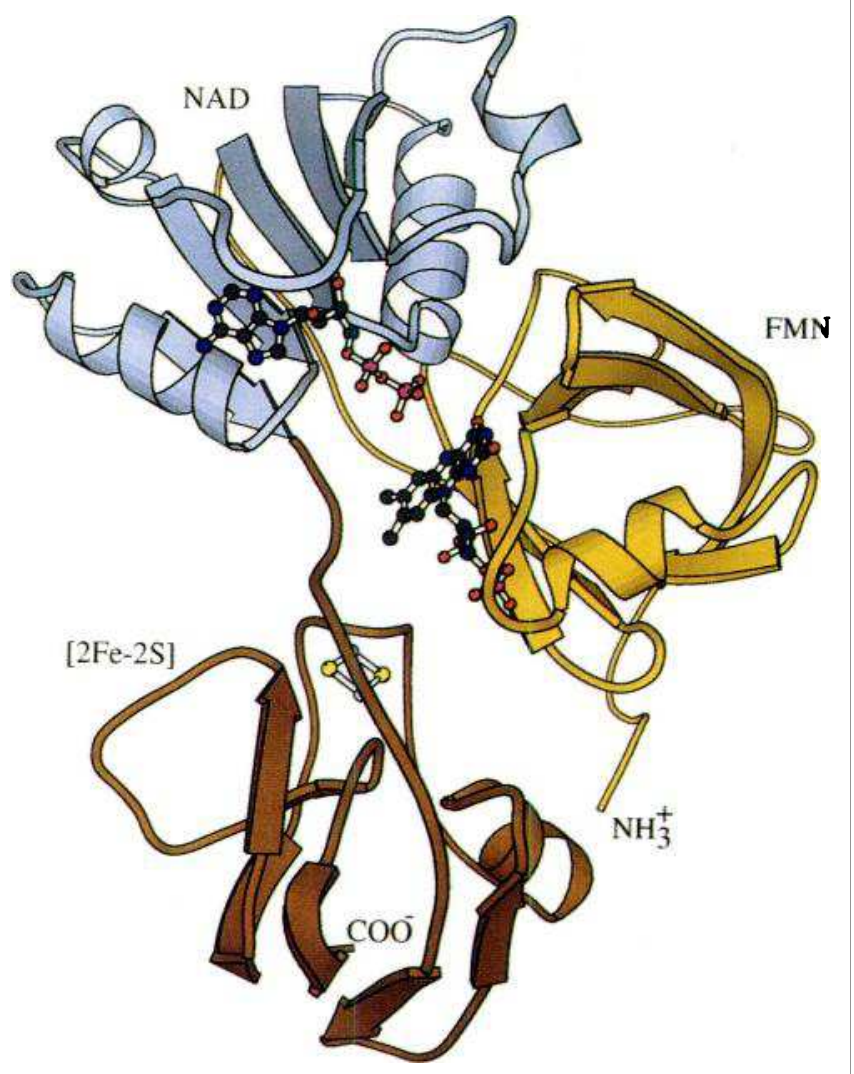

Figure 2. A drawing of PDR with NAD+ bound: FMN is associated primarily with the $\mathrm{NH}_{2}$-terminal domain, and pyridine nucleotide with the central domain. The substrate and prosthetic groups are brought together at the interface between the domains. This view emphasizes the modular nature of the enzyme and shows the linker attaching the [2Fe-2S] domain to the rest of the protein.

reductase (22) and cytochrome $b_{5}$ reductase (23), which have been recently described, confirm that these proteins are structural relatives of FNR. Considerable variation exists in the domain connectivities of bacterial dioxygenase reductases belonging to the FNR family. Vanillate de- methylase oxidoreductase (24) is organized like PDR with a $\mathrm{COOH}$-terminal ferredoxin domain. In contrast, the reductase components of the naphthalene (25) and benzoate (26) dioxygenase systems have $\mathrm{NH}_{2}$-terminal ferredoxin domains. As can be seen in Fig. 2, addition of several residues at the $\mathrm{COOH}$ terminus of the ferredoxin domain could link the [2Fe-2S] domain to the $\mathrm{NH}_{2}$ terminus rather than to the $\mathrm{COOH}$ terminus of PDR while still preserving the overall structure and interfaces of the three domains (27). The observed variation in domain connectivity among the iron-sulfur flavoprotein reductases of the FNR family supports the idea that these enzymes have evolved by gene fusions.

\section{Flavin and pyridine nucleotide binding sites in PDR and FNR}

Despite the sequence and structural similarities between FNR and PDR, these proteins have different pyridine nucleotide and flavin binding specificities. FNR binds FAD and NADP(H), whereas PDR binds FMN and NAD(H). The flavin nucleotide binding specificity is defined in part by residues preceding the major $\alpha$-helix of the FMN binding domain. In PDR, arginine80 in the sequence (G-R-G-G-S) binds to the ribityl phosphate of FMN (Fig. 3). In the corresponding phosphate binding sequence (K-G-V-C-S) in FNR (based on structure alignment), the arginine is replaced with glycine-130 (19). Comparison of the flavin binding sites of PDR and FNR suggests that the arginine side chain would interfere sterically with the binding of the adenosine phosphate portion of FAD. This sequence is therefore an important determinant of flavin binding specificity.

Structural features have been identified that are critical to the preferential binding of NADH to PDR and NADPH to FNR. The $2^{\prime}$-phosphate of the adenine portion of NADP ${ }^{+}$is bound to FNR through interactions with $\mathrm{Ser}^{234}$ and $\mathrm{Arg}^{235}$. $\mathrm{Ser}^{234}$ is replaced in PDR by Asp ${ }^{173}$ (Fig. 3), which hydrogen bonds directly to the $2^{\prime}-\mathrm{OH}$ of NADH (18). Residues interacting with the 2 '-phosphate of NADPH were first identified in glutathione reductase as the primary structural features that discriminate between NADH and NADPH (20).
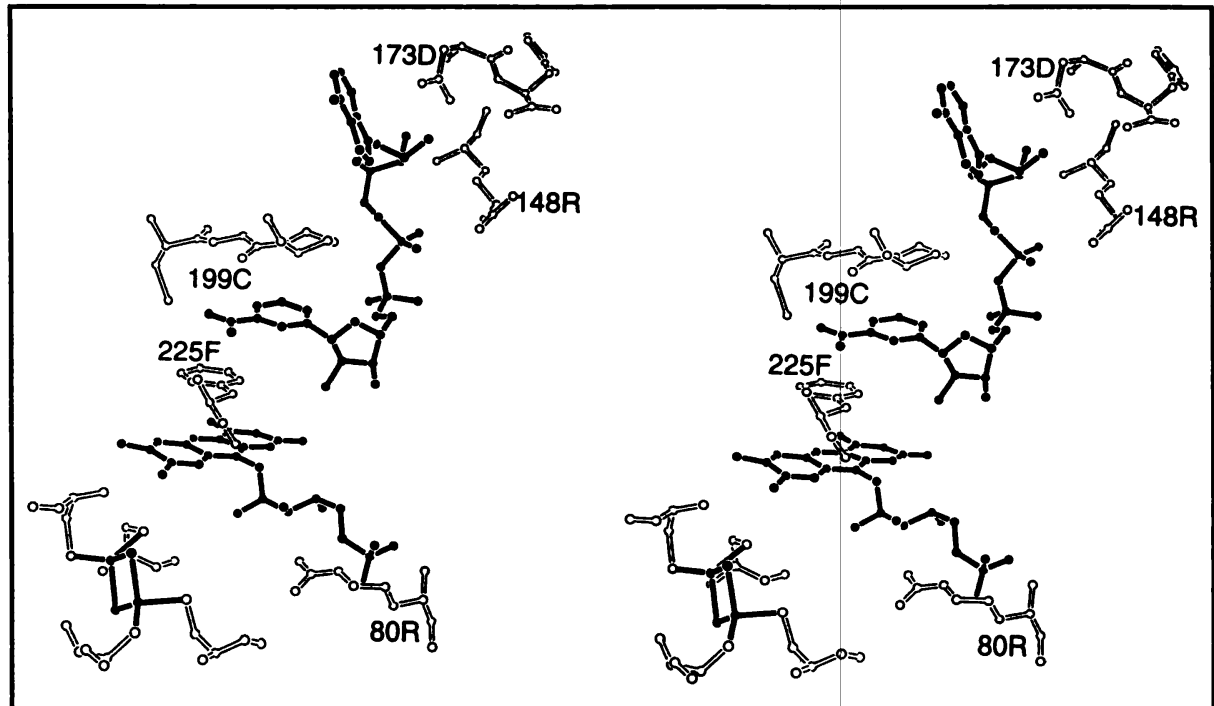

Figure 3. A drawing showing the mode of binding of NADH to $\mathrm{PDR}_{2 \mathrm{e}}$ : the prosthetic groups and cofactor are shown with black bonds and the protein with gray bonds. In this complex, whichmay correspond to the intermediate MC-3 (Scheme 1), the ring of $\mathrm{Phe}^{225}$ lies between the flavin and the nicotinamide. Side chains stabilizing the $\mathrm{NADH}\left(\mathrm{Asp}^{173}, \mathrm{Arg}^{148}\right)$ and $\mathrm{FMN}_{\mathrm{sq}}$ $\left(\mathrm{Arg}^{\mathrm{BO}}\right)$ are visible. 


\section{Domain interfaces}

The tri-lobed structure of PDR is held together primarily by interactions at two major interfaces, one between the FMN and NADH domains and the other between the FMN and [2Fe-2S] domains. Interactions between the NADH and iron-sulfur domains may nevertheless be important for catalysis (see later text). The contacts connecting the FMN and NADH binding domains of PDR are typical for interdomain surfaces, with a large number of hydrophobic van der Waals contacts and interdigitating aromatic side chains. In contrast, the interface between the $[2 \mathrm{Fe}-2 \mathrm{~S}]$ and FMN binding domains includes salt bridges, direct interdomain hydrogen bonds, and indirect hydrogen bonding through bridging water molecules. These contacts are more typical of surfaces where water-soluble polypeptides associate (18). The nature of the interface and the structural similarities of PDR to ferredoxin $\mathrm{NADP}^{+}$reductase and ferredoxin suggest that both the ferredoxin and FNR-like portions of PDR may be able to exist as separate entities. Indeed, we have been able to produce a truncated form of PDR that lacks an iron-sulfur domain.

\section{TRUNCATED PDR}

PDR can be cleaved selectively at residue 229 in the linker that attaches the $[2 \mathrm{Fe}-2 \mathrm{~S}]$ domain to the NADH binding domain (Fig. 2) (28). Presumably the backbone solvent accessibility and disorder of this region (16) make it an especially good target for proteolytic cleavage. The [2Fe-2S]-containing peptide is destroyed by proteolysis after its initial release from PDR; however, the FNR-like domain of PDR is recovered in high yield. This proteolysis can be monitored spectrally by observing the loss of the iron-sulfur component of the spectrum and the formation of a spectrum that closely resembles that of FNR. FMN remains tightly bound to the surviving $25 \mathrm{kDa}$ peptide, which is stable at $4^{\circ} \mathrm{C}$ for several days and has been extensively characterized. This truncated form of PDR is referred to as PDR(-FeS). The ability of PDR(-FeS) to retain the FMN prosthetic group is consistent with the crystal structure of PDR; only one residue from the [2Fe-2S] domain, $\mathrm{Ser}^{274}$, interacts directly with FMN (27). $\mathrm{NH}_{2}$-terminal amino acid sequence analysis shows that $\mathrm{PDR}(-\mathrm{FeS})$ retains the entire $\mathrm{NH}_{2}$ terminus of holo PDR. Accurate determination of the molecular weight of PDR(-FeS) by electrospray mass spectroscopy identifies the cleavage site in the linker region as the bond linking $\mathrm{Asn}^{229}$ and $\mathrm{Thr}^{230}$ in the subsequence T-N-T. Despite the loss of the $10 \mathrm{kDa}$ [2Fe-2S] domain, $\mathrm{PDR}(-\mathrm{FeS})$ retains many properties of the holoenzyme as discussed later.

\section{SPECTROSCOPIC AND REDOX PROPERTIES OF PDR}

Both the plant-type [2Fe-2S] center and the FMN cofactor contribute to the visible absorbance spectrum of PDR. The spectrum of PDR(-FeS) is representative of the flavin component and is quite similar to the spectrum of ferredoxin$\mathrm{NADP}^{+}$reductase. The ferredoxin component of the PDR spectrum can be calculated from the difference of the spectra of PDR and of PDR(-FeS) (see Fig. 5A) and closely resembles the absorbance spectrum of adrenodoxin (29).

\section{Redox potentials}

Reductive titrations of PDR with NADH or dithionite proceed in two stages. In the first phase, the iron-sulfur and FMN moieties appear to react simultaneously, giving rise to a form of the enzyme containing a reduced iron-sulfur center and a flavin semiquinone (SQ). The maximum semiquinone concentration reached during this part of the titration is $\sim 80 \%$ of the total enzyme concentration. The [2Fe-2S] and $\mathrm{FMN}_{\mathrm{ox} / \mathrm{sq}}$ redox couples are closely matched (both about $-174 \mathrm{mV}$ at $\mathrm{pH} \mathrm{7)}$. In the second phase of the titration, the SQ form of PDR is converted to the threeelectron reduced enzyme (see spectra representative of MC-3 and PDR 3 e- in Fig. 4). The FMN sq/hq $_{\text {sq }}$ couple, with a midpoint potential of $-287 \mathrm{mV}$ at $\mathrm{pH} 7$, is well resolved from the $F M N_{o x / s q}$ couple. The $F M N_{o x / s q}$ potential of $\mathrm{PDR}(-\mathrm{FeS})$ is the same as the $\mathrm{FMN}_{\mathrm{ox} / \mathrm{sq}}$ couple of PDR (28). However, during the reduction of PDR(-FeS), a maximum of only $\sim 60 \%$ of the total enzyme is stabilized as semiquinone; this diminished level of semiquinone is due to an increase in the $\mathrm{FMN}_{\mathrm{sq} / \mathrm{hq}}$ potential to $-235 \mathrm{mV}$, about 50 $\mathrm{mV}$ more positive than the $\mathrm{FMN}_{\mathrm{sq} / \mathrm{hq}}$ couple of PDR. It is possible that the negative charge $(-3)$ associated with the reduced [2Fe-2S] center of PDR interacts with the anionic flavin hydroquinone to decrease the $\mathrm{FMN}_{\mathrm{sq}} / \mathrm{hq}$ midpoint potential in PDR relative to that of PDR(-FeS), where the iron-sulfur cluster is absent.

The midpoint potentials of PDR favor spontaneous electron transfer from NADH to FMN to [2Fe-2S] (Fig. 1). This flow of electrons is opposite to the direction of electron transfer in the ferredoxin-NADP ${ }^{+}$reductase system, where two electrons from ferredoxin are passed sequentially to FAD and subsequently transferred as a hydride to NADP ${ }^{+}$. The redox potentials of $\mathrm{FNR} \mathrm{E}_{\mathrm{m}, 7}\left(\mathrm{FMN}_{\mathrm{ox} / \mathrm{hq}}=-360 \mathrm{mV}\right.$ (30) facilitate formation of NADPH from reduced ferredoxin. Efforts have been made to correlate differences in protein structure in the region of the [2Fe-2S] binding sites of PDR and ferredoxin to differences in the redox potential of these sites (18). Calculations of electrostatic interactions suggest that hydrogen bonds to the cysteine and inorganic sulfide ligands of the [2Fe-2S] center of PDR are the most important structural features contributing to the relatively high redox potential of this center compared with that of ferredoxin.

\section{EPR spectroscopy}

The EPR spectrum of two-electron reduced PDR has g-values at $2.041,1.949$, and 1.900 , representative of a [2Fe$2 \mathrm{~S}$ ] center, and 2.008 , corresponding to the neutral blue flavin semiquinone (31). Strong electronic interactions between the paramagnetic $[2 \mathrm{Fe}-2 \mathrm{~S}]_{\mathrm{red}}$ and $\mathrm{SQ}$ radical would be expected because these centers are only $4.7 \AA$ apart at their closest approach. It is therefore surprising that the spectra of frozen solutions approximate the sum of the 

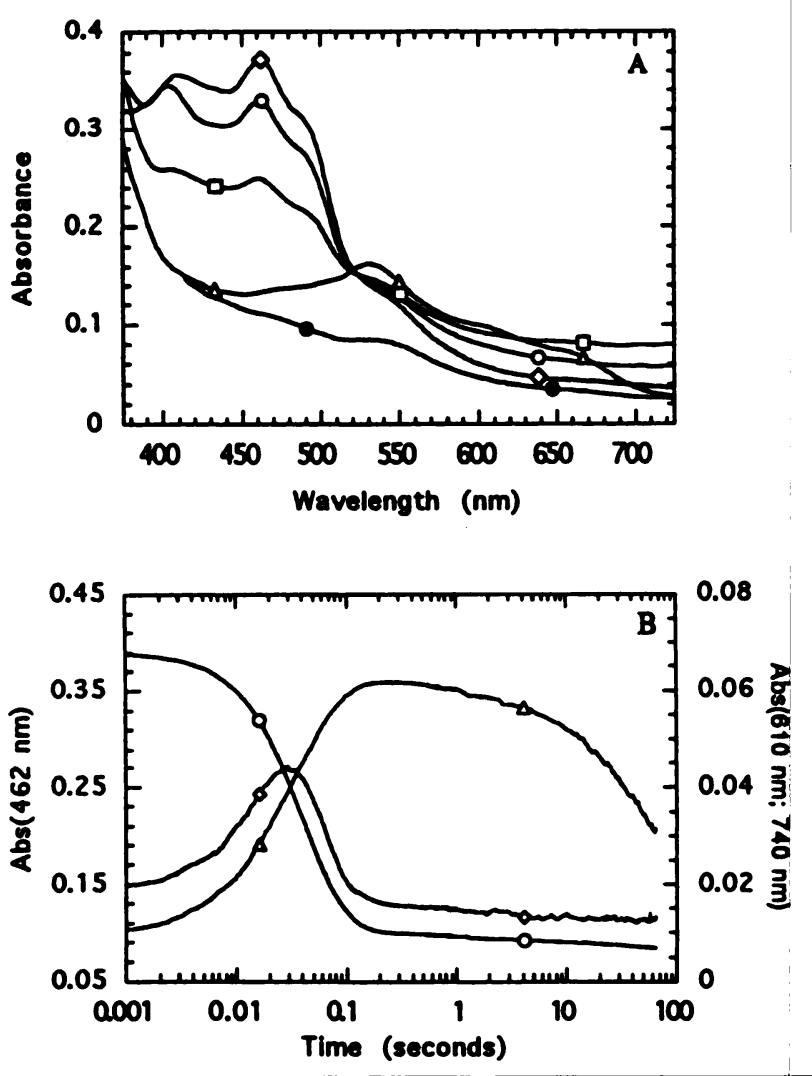

Figure 4. Spectra of kinetic intermediates in the reaction of PDR with NADH: $A$ ) oxidized PDR $(\diamond)$; diode array spectrum representative of CT-1 (O); diode array spectrum representative of CT* $(\square)$; spectrum representative of MC-3 $(\Delta)$; spectrum representative $\left.\operatorname{PDR}_{3 e-}(O) . B\right)$ Kinetics of the reaction of PDR with NADH monitored at $462 \mathrm{~nm}(\mathrm{O})$, $610 \mathrm{~nm}(\Delta)$, and $740 \mathrm{~nm}(\diamond)$. Formation of CT-1 is most evident between $2 \mathrm{~ms}$ and $10 \mathrm{~ms}$ at $740 \mathrm{~nm}$ and $610 \mathrm{~nm}$. The maximum concentration of

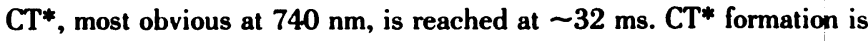
paralleled by hydride transfer and flavin reduction, observed as a decrease in absorbance at $462 \mathrm{~nm}$. NAD release occurs with a loss of CT* absorbance at $740 \mathrm{~nm}$. There is a simultaneous increase in absorbance at $610 \mathrm{~nm}$, which reports the formation of MC-3. Disproportionation of PDR to form PDR $_{3 e}$ - is detected as a further decrease in $610 \mathrm{~nm}$ absorbance beginning at about $1 \mathrm{~s}$ after the mixing of reactants.

spectra of isolated paramagnetic species (R. W. Dunham, personal communication). Preliminary simulations of these spectra employing the "magic angle" between the FMN and [2Fe-2S] center can account for some of the results (32). Studies are currently under way to characterize the electronic interactions in this system (33).

\section{REACTION OF PDR WITH PYRIDINE NUCLEOTIDES}

The natural substrate for PDR is NADH, although NADPH will slowly reduce PDR. This is consistent with the presence of Asp ${ }^{173}$ and the other structural features conferring specificity for NADH (discussed previously). Product formation during the steady-state reaction of the phthalate dioxygenase system occurs with a broad $\mathrm{pH}$ optimum in the $\mathrm{pH}$ range $6.8-8.2$. At $4^{\circ} \mathrm{C}$, the maximum steady-state reaction velocity with $\mathrm{PDO}$ as an electron acceptor is $12 \mathrm{NADH}$ oxidized/s; $K_{\mathrm{m}}(\mathrm{NADH})$ is $10 \mu \mathrm{M}$ and $K_{\mathrm{m}}$ (PDO) is $\sim 1.4$ $\mu \mathrm{M}$ (34). This latter $K_{\mathrm{m}}$ value is close to the dissociation constant of the PDO-PDR complex $(\sim 1 \mu \mathrm{M})$, calculated from changes in flavin fluorescence that occur during the titration of PDO with PDR (35).

We have used stopped flow spectrophotometry in a study of the kinetics of the reductive half-reaction of PDR with pyridine nucleotides $(28,29)$. These investigations have identified the intermediates of Scheme 1 and have determined the reaction rate constants included in that scheme. NADH first binds to oxidized PDR, forming a primary Michaelis complex (MC-1). This process is a rapid equilibrium with respect to subsequent steps in the reduction reaction, and it is complete within the mixing time of the instrument $(\sim 2 \mathrm{~ms})$. Because $\mathrm{NAD}^{+}$binds about two orders of magnitude more weakly than does NADH to oxidized PDR, only a small amount of MC-2 (Scheme 1) forms when $\mathrm{NAD}^{+}$and $\mathrm{NADH}$ are present at equal concentrations.

The first observed kinetic phase $\left(116 \mathrm{~s}^{-1}\right)$ is signaled by a slight decrease in absorbance at wavelengths shorter than $500 \mathrm{~nm}$ and an increase in absorbance beginning at $\sim 550$ $\mathrm{nm}$ and extending beyond $800 \mathrm{~nm}$ (Fig. $4 A$ and Fig. $4 B$ ). These changes are consistent with the formation of a charge-transfer complex between NADH and oxidized FMN (CT-1 in Scheme 1). The analogous intermediate observed in the reaction of PDR(-FeS) with NADH shows the spectral features of this complex in the absence of the absorbance contribution from the oxidized iron-sulfur center (Fig. 5C). The intermediate CT-1 is spectrally quite similar to the charge-transfer complex formed between

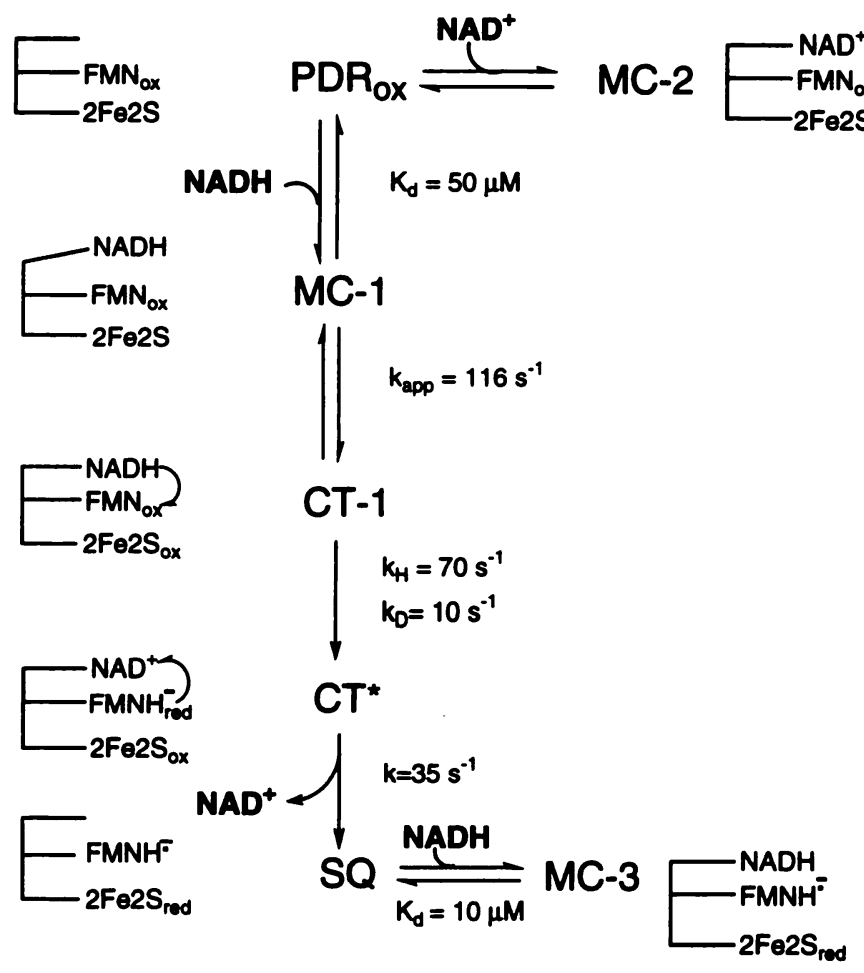

Scheme 1. Mechanism of the reductive half-reaction of PDR with NADH. Stick diagrams detail the redox and complexation state of intermediate species in the reaction (crystal structures for $\mathrm{PDR}_{\mathrm{ox}}, \mathrm{MC}-2$, and MC-3 have been determined). 
NADPH and oxidized FNR (36).

The second kinetic phase is consistent with the reduction of the flavin moiety and the formation of a charge-
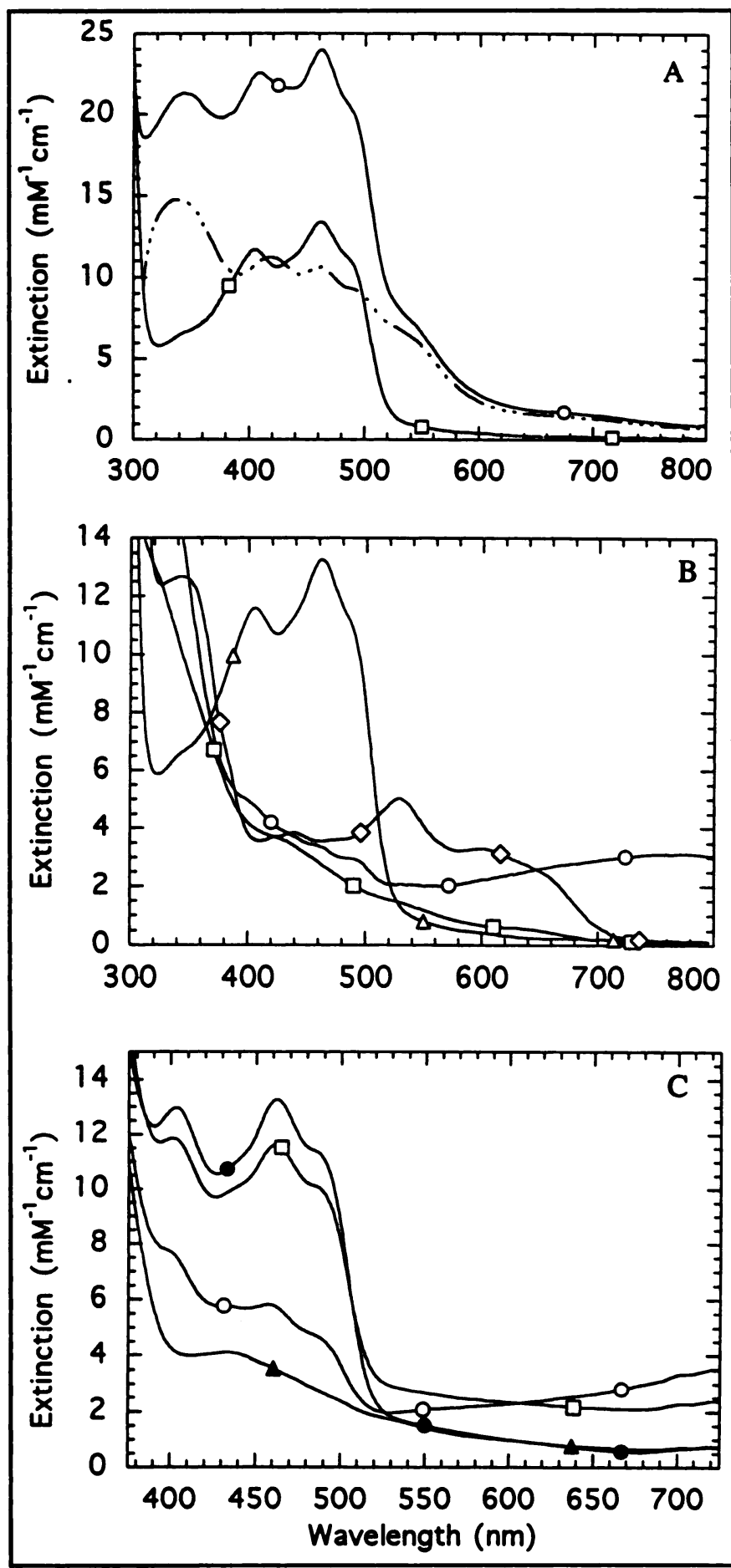

Figure 5. Components of the phthalate dioxygenase system: $A$ ) absorbance spectra of oxidized PDR $(O)$ and PDR(-FeS) ( $\square)$; difference spectrum representative of the $[2 \mathrm{Fe}-2 \mathrm{~S}]$ component of the PDR absorbance spectrum, generated by subtraction of the spectrum of PDR (-FeS) from PDR (-• - - ). B) Spectra of oxidized PDR (-FeS) ( $\triangle$ ); PDR $(-\mathrm{FeS})$ semiquinone, generated by reductive titration $(\diamond)$; PDR $(-\mathrm{FeS})$ MC-4 (charge-transfer complex of FMN ${ }_{\text {red }}$ with NAD) (O); two-electron reduced PDR (-FeS) ( $\square$ ). C) Spectra of intermediates in the reaction of PDR (-FeS) with pyridine nucleotides; oxidized PDR (-FeS) (O); CT-1'

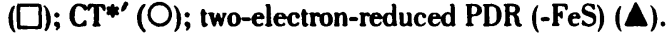

transfer complex between $\mathrm{FMNH}^{-}$and $\mathrm{NAD}^{+}\left(\mathrm{CT}^{*}\right.$ in Scheme 1). It is observed as a further increase in absorbance in the long wavelength region and a large decrease in absorbance between 300 and $500 \mathrm{~nm}$. The spectrum of the analogous intermediate occurring in the reductive halfreaction of $\mathrm{PDR}(-\mathrm{FeS})$ is shown in Fig. $5 B$. The spectrum of the CT* species is similar to that of the charge-transfer complex formed between $\mathrm{NADP}^{+}$and the reduced FAD of adrenodoxin reductase (37). When NADD is substituted for NADH in the reduction reaction, the rate of formation of CT* is decreased from $70 \mathrm{~s}^{-1}$ to $10 \mathrm{~s}^{-1}$. This sevenfold deuterium isotope effect identifies this reaction phase as hydride transfer.

After hydride transfer, $\mathrm{NAD}^{+}$is released from $\mathrm{PDR}$ (Scheme 1). This phase of the reaction (35 s $\mathrm{s}^{-1}$ in PDR) is observed as a decrease of the charge-transfer absorbance at wavelengths greater than $700 \mathrm{~nm}$, an increase in absorbance at wavelengths between $520 \mathrm{~nm}$ and $650 \mathrm{~nm}$, and a decrease in absorbance below $520 \mathrm{~nm}$ (Fig. 4). The loss of the charge-transfer absorbance represents $\mathrm{NAD}^{+}$release, and the increases in the 500-600 $\mathrm{nm}$ region are consistent with the formation of a neutral blue flavin SQ that results from intramolecular electron transfer. Small decreases in absorbance in the $400-500 \mathrm{~nm}$ region result from a combination of flavin oxidation to semiquinone and [2Fe-2S] reduction (Fig. $4 A, B$ ). This semiquinone species does not occur in the reductive half-reaction of PDR(-FeS) with NADH. (The spectrum of the flavin semiquinone of PDR(-FeS), generated by reductive titration, is presented in Fig. $5 B$.) Because $\mathrm{NAD}^{+}$release, flavin oxidation to semiquinone, and iron-sulfur reduction are observed to occur as a single kinetic phase with PDR, it is unclear which one of these events controls this phase of the reaction.

As noted, PDR(-FeS) proceeds through the same types of flavin-pyridine nucleotide intermediates that are observed in the reductive half-reaction of PDR. However, after $\mathrm{NAD}^{+}$release from PDR(-FeS), both hydride electrons remain localized on the flavin (compare Fig. $5 C$ and Fig. 4A). Titration of this reduced form of $\mathrm{PDR}(-\mathrm{FeS})$ with $\mathrm{NAD}^{+}$results in the formation of a charge-transfer complex (Fig. $5 B$ ) that spectrally resembles the flavin component of CT* formed in the reductive half-reaction of PDR. This species presumably involves a direct stacking interaction of the reduced flavin and $\mathrm{NAD}^{+}$in a complex similar to the complex of NADPH with reduced glutathione reductase $(20,27)$.

\section{Structures related to intermediates in the reductive half-reaction}

Crystal structures of oxidized PDR in complex with $\mathrm{NAD}^{+}$ or $\mathrm{AAD}^{+}$, of two-electron reduced $\mathrm{PDR}$ in complex with

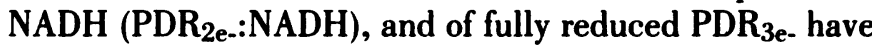
been determined $(16,38)$. The nicotinamide ring does not contact the flavin ring in any of the crystallographically observed pyridine nucleotide complexes. When $\mathrm{NAD}^{+}$or $\mathrm{AAD}^{+}$is bound to PDR, the nicotinamide ring cannot be discerned, implying that it is mobile. The crystal structure

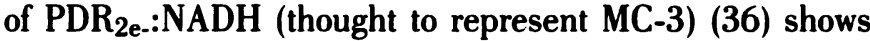




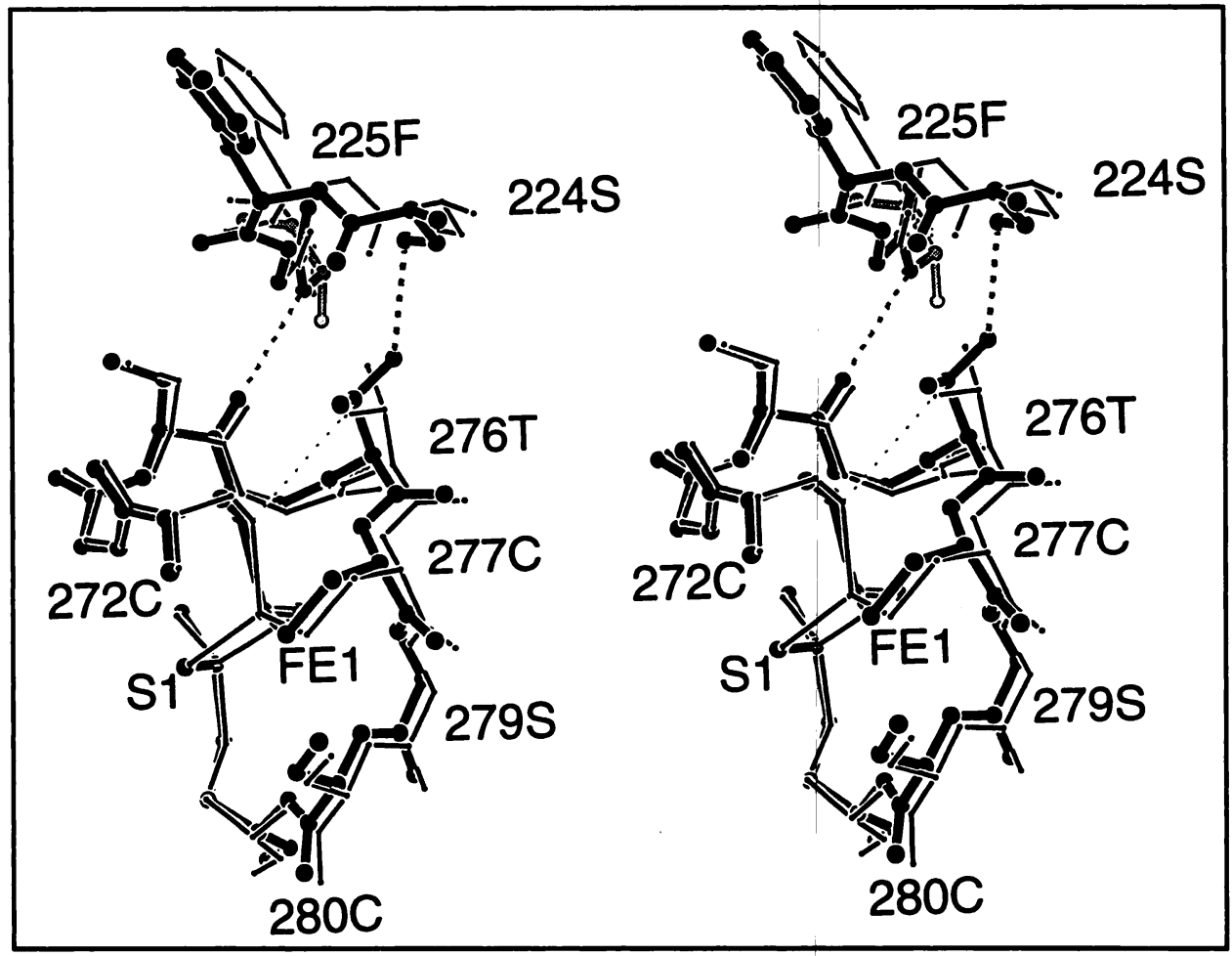

Figure 6. Local changes near the iron-sulfur center that accompany formation of the species $\mathrm{PDR}_{2 e}: \mathrm{NADH}$. Ligand-free oxidized PDR is shown with thin bonds and PDR $_{2 e}$ : NADH with thick bonds. the loop including residues 273-279 readjusts when nucleotide binds. In PDR $_{2 \mathrm{e} \text { : }} \mathrm{NADH}$, alterations in interdomain contacts are observed that show how pyridine nucleotide binding may affect the environment of the [2Fe-2S] cluster. Residues 224-227, at the $\mathrm{COOH}$ terminus of the $\mathrm{NAD}^{+}$domain, move toward the iron-sulfur cluster and two new interdomain hydrogen bonds are formed. One connects the backbone NH 227 of the NAD ${ }^{+}$ domain to carbonyl 0 of 274 of the [2Fe-2S] domain and the other connects Oy of $\mathrm{Ser}^{224}$ with $0 \gamma$ of $\mathrm{Thr}^{276}$ from the iron-binding loop. In order to make the second hydrogen bond, the $\mathrm{Thr}^{276}$ side chain rotates, breaking the interaction between $\mathrm{Thr}^{276}$ and Cys Sy 272 , an iron ligand. These subtle rearrangements in the interactions between the NAD and [2Fe-2S] domains of the MC-3 type complex may provide thermodynamic linkage among the redox centers of PDR (39).

the benzene ring of $\mathrm{Phe}^{225}$ intervening between the flavin and nicotinamide rings (Fig. 3); as expected, this species shows no charge-transfer absorbance. In the oxidized structure of glutathione reductase, $\mathrm{Tyr}^{197}$ intervenes similarly, and it has been shown that when NADPH binds, $\mathrm{Tyr}^{197}$ moves to permit direct interaction between the pyridine nucleotide and the flavin (20). Thus, during catalysis it might be expected that $\mathrm{Phe}^{225}$ of PDR moves to permit the nicotinamide to come in contact with the isoalloxazine ring of the flavin. Such interaction is required for both charge-transfer and hydride transfer.

\section{Regulation of intramolecular electron transfer in PDR}

The crystal structure of PDR indicates that the flavin and iron-sulfur centers are separated by an edge-to-edge distance of only $4.7 \AA$ (16). The driving force favoring electron transfer from fully reduced flavin to [2Fe-2S] is approximately $100 \mathrm{mV}(30) .{ }^{5}$ Based on the contributions of distance and thermodynamic driving force $(39,40)$, the intramolecular electron transfer from FMN to [2Fe-2S] in PDR is predicted to be several orders of magnitude faster than the observed rate of $35 \mathrm{~s}^{-1}$. In PDR(-FeS), the rate of $\mathrm{NAD}^{+}$release closely matches the rate of intramolecular

\footnotetext{
${ }^{5}$ The difference in midpoint potential between the SQ/PDR $\mathrm{P}_{\mathrm{x}}$ and $\mathrm{PDR}_{3 \mathrm{e}} / \mathrm{SQ}$ couples provides an estimate of the thermodynamic driving force for a single electron transfer from the flavin hydroquinone of the $\mathrm{CT}^{*}$ intermediate to the oxidized [ $2 \mathrm{Fe}-2 \mathrm{~S}$ ] center. However, this is likely to be an overestimation, because an interaction of the negatively charged, reduced iron-sulfur center in $\mathrm{PDR}_{3 \mathrm{e}}$ may decrease the $\mathrm{FMN}_{\mathrm{hq} / \mathrm{sq}}$ couple of $\mathrm{PDR}_{3 \mathrm{se}}$ relative to the $\mathrm{FMN}_{\mathrm{hq} / \mathrm{sq}}$ couple of $\mathrm{CT}^{*}$ in which the iron center is oxidized.
}

electron transfer and $\mathrm{NAD}^{+}$release in PDR. This supports the idea that the release of $\mathrm{NAD}^{+}$controls the rate of intramolecular electron transfer; it is inconsistent with intramolecular electron transfer controlling the rate of $\mathrm{NAD}^{+}$ release. Thus, structural changes effected by the $\mathrm{NAD}^{+}$ release may control the electron transfer by contributing to the reorganization energy (39) and/or by gating the transfer directly (41).

Crystal structures of reduced and ligated states of PDR show changes in the vicinity of the [2Fe-2S] center that are linked to reduction and to pyridine nucleotide binding $(16$, 38 ) and may reflect the reorganization predicted by the Marcus theory (39) (Fig. 6). Hydrogen bonds to the cysteine ligands of the [2Fe-2S] center appear to shorten, and the geometry of $\mathrm{Fe}-\mathrm{l}$ (the iron of the [2Fe-2S] center closest to the FMN moiety) is distorted toward trigonal bipyramidal (38).

\section{CONCLUSIONS}

PDR illustrates some of the complexities of how hydride and single electron carriers are coupled in biological systems. Scheme 1 shows that in PDR at least five species are involved in this process. The structures of some of these species have been determined and support the kinetic scheme. Structural information is not yet available for the kinetically important charge-transfer intermediates CT-1 and CT*, which occur only transiently in the reductive half-reaction. Pyridine nucleotide complexes with PDR that have been observed so far do not show the nicotinamide ring interacting with the isoalloxazine of the flavin as required for both charge-transfer interactions and hydride transfer. However, changes observed in glutathione reductase, oxidized or reduced in complex with NADPH, provide a model for the sequence of events that must ac- 
company pyridine nucleotide binding and hydride transfer in PDR. Presumably the binding of NADH to PDR triggers a displacement of $\mathrm{Phe}^{225}$ to a position that allows the nicotinamide ring to interact transiently with the flavin for hydride transfer.

Another aspect in this catalysis is the low rate of intramolecular electron transfer from reduced FMN to the [2Fe2S] center. Electron transfer theory (39) predicts very rapid internal electron transfer in PDR (CT* to SQ in Scheme 1), but the rate is $35 \mathrm{~s}^{-1}$. The small structural changes that occur upon reduction (Fig. 6) do not seem sufficient to account for this phenomenon. It appears instead that the release of $\mathrm{NAD}^{+}$may be a more important event in the control of electron transfer to the [2Fe-2S] center. It remains to be determined how this regulation occurs and what significance slow electron transfer might have in the phthalate dioxygenase system.

The authors would like to thank Drs. V. Massey and Lihua Wang for helpful discussions. Support for these studies has been provided by National Institutes of Health grant GM20877 to D.P.B. and GM16429 to M.L.L.

\section{REFERENCES}

1. Batie, C. J., Ballou, D. P., and Correll, C. C. (1992) Phthalate dioxygenase reductase and related iron-sulfur containing electron transferases. In Chemistry and Biochemistry of Flavoenzymes, Vol. 3 (Müller, F., ed) pp. 543-556, CRC Press, Boca Raton, Florida

2. Mason, J. R., and Cammack, R. (1992) The electron transport proteins of hydroxylating bacterial dioxygenases. Annu. Rev. Microbiol. 46, 277-305

3. Gurbiel, R. J., Batie, C. J., Sivaraja, M., True, A. E., Fee, J. A., Hoffman, B. M., and Ballou, D. P. (1989) Electron-nuclear double resonance spectroscopy of ${ }^{15} \mathrm{~N}$-enriched phthalate dioxygenase from Pseudomonas cepacia proves that two histidines are coondinated to the [2Fe-2S] Rieske-type clusters. Biochemistry 28, 4861-4871

4. Batie, C. J., LaHaie, E., and Ballou, D. P. (1987) Purification of phthalate oxygenase and phthalate oxygenase reductase from Pseudomonas cepacia. J. Biol. Chem. 262, 1510-1518

5. Yamaguchi, M., and Fujisawa, H. (1980) Purification and characterization of an oxygenase component in the benzoate 1,2-dioxygenase system from Pseudomonas ariilla C-1. J. Biol. Chem. 255, 5058-5063

6. Locher, H. H. Leisinger, T., and Cook, A. M. (1991) 4-Toluene sulfonate methyl monooxygenase from Comamonas testosteroni 1-2: purification and some properties of the oxygenase component. J. Bacteriol. 173, 3741-3748

7. Schweizer, D., Markus, A., Seez, M., Ruf, H. H., and Lingens, F. Purification and some properties of component b of the 4-chlorophenylacetate 3,4-dioxygenase from Pseudomonas species strain CBS3. J. Biol. Chem. 262, 9340-9346

8. Fetzner, S., Muller, R., and Lingens, F. (1992) Purification and some properties of 2-halobenzoate 1, 2-dioxygenase, a two-component enzyme system from Pseudomonas cepacia 2CBS. J. Bacteriol. 174, 279-290

9. Axcell, B. C., and Ceary, P. J. (1975) Purification and some properties of a soluble benzene oxidizing system from Pseudomonas putida. Biochem. J. 146, 173-183

10. Sauber, K., Frohner, C., Rosenberg, G., and Eberspacher, J. (1973) Purification and Properties of pyrazon dioxygenase from pyrazon degrading bacteria. Eur. J. Biochem. 741, 89-97

11. Bunz V. B., and Cook, A. M. (1993) Dibenzofuran 4,4a-dioxygenase from Sphingomonas sp. strain RW 1: angular dioxygenation by a three-component enzyme system. J. Bacteriol. 175, 6467-6465

12. Yeh, W. K., Gibson, D. T., and Liu, T. N. (1977) Toluene dioxygenase: a multicomponent enzyme system. Biochem. Biophys. Res. Commun. 78, 401-410

13. Haigler, B. E., and Gibson, D. T. (1990) Purification and properties of NADH-ferredoxin reductase, a component of naphthalene dioxygenase from Pseudomonas sp. strain NCIB 9816. J. Bacteriol. 172, 457-468

14. Suen, W., and Gibson, D. T. (1993) Isolation and characterization of the subunits of the terminal subunits of naphthalene dioxygenase from Pseudomonas putida NCIB 9816-4. J. Bacteriol. 175, 5877-5881

15. Romanov, V., and Hausinger, R. P. (1994) Pseudomonas aeruginosa 142 uses a three-component ortho-halobenzoate 1, 2-dioxygenase for metabolism of 2, 4-dichloro- and 2-chlorobenzoate. J. Bacteriol. 176, 3368-3374

16. Correll, C. C., Batie, C. J., Ballou, D. P., and Ludwig, M. L. (1992) Phthalate dioxygenase reductase: a modular structure for electron transfer from pyridine nucleotides to [2Fe-2S]. Science 258, 1604-1610

17. Rossman, M. G., Moras, D., and Olsen, K. W. (1974) Chemical and biological evolution of a nucleotide-binding protein. Nature (London) 250, 194-199

18. Correll, C. C., Ludwig, M. L., Bruns, C. M., and Karplus, P.A. (1993) Structural Prototypes of an extended family of navoprotein reductases: comparison of phthalate dioxygenase reductase with ferredoxin reductase and ferredoxin reductase and ferredoxin. Protein Sci. 12, 2112-2133

19. Karplus, P. A., Daniels, M. J., and Herriott, J. R. (1991) Atomic structure of ferredoxin-NADP ${ }^{+}$reductase: pmototype for a structurally novel flavoenzyme family. Science 251, 60-66

20. Pai, E. F., Karplus, P. A., and Schultz, C. E. (1988) Crystallographic analysis of the binding of NADPH, NADPH fragments, and NADPH analogues to glutathione reductase. Biochemistry 27, 4465-4474

21. Andrews, S. C., Shipley, D., Keen, J. N., Findlay, J. B. C., Harrison, P. M., and Guest, J. R. (1992) The haemoglobin-like protein (HMP) of Escherichia coli has ferrisiderophore reductase activity and its C-terminal domain shares homology with ferredoxin $\mathrm{NADP}^{+}$reductases. FEBS Lett. 302, 247-252

22. Lu, G., Campbell, W.H., Schneider, C. and Lindqvist, Y. (1994) Crystal structure of the FAD-containing fragment of corn nitrate reductase at $2.5 \AA$ resolution: relationship to other flavoprotein reductases. Structure 2 , 809-821

23. Takano, T., Bando, S., Horii, C., Higashiyama, M., Ogawa, K., Sato, M., Katsuya, Y., Danno, M., Yubisui, T., Shirabe, K., and Takeshita, M. (1993) The structure of human erythrocyte $\mathrm{NADH}$-cytochrome $b_{5}$ reductase at 2.5 $\AA$ resolution. In Flavins and Flavoproteins 1993 (Yagi, K., ed) pp. 407-412 Walter de Gruyter and Co., Berlin

24. Brunel, F., and Davison, J. (1988) Cloning and sequencing Pseudomonas genes containing vanillate demethylase. J. Bacteriol. $170,4924-4930$

25. Simon, M. J., Osslund, T. D., Saunders, R., Ensley, B. D., Suggs, S., Harcourt, A., Suen, W. C., Cruden, D. L., Gibson, D. T., and Zylstra, G. J. (1993) Sequences encoding naphthalene dioxygenases in Pseudomonas putida strains 67, and NCIB 9816-4. Gene 127, 31-37

26. Neidle, E. L., Hartnett, C., Ornston, L. N., Bairoch, A., Rekik, M., and Harayama, S. (1991) Nucleotide sequences of the Acinetobacter calcoaceticus benABC genes for benzoate-1,2-dioxygenase reveal evolutionary relationships among multicomponent oxygenases. J. Bacteriol. 173, 5385-5395

27. Correll C. C. (1992) Structure determination and analysis of an iron-sulfor flavoprotein: phthalate dioxygenase reductase. Ph.D. dissertation, The University of Michigan, Ann Arbor

28. Gassner, G., and Ballou, D.P. (1995) Preparation and characterization of a truncated form of phthalate dioxgenase reductase that lacks an iron-sulfer domain. Biochemistry In press

29. Gassner, G., Wang, L. Batie, C., and Ballou, D. P. (1994) Reaction of phthalate dioxygenase reductase with $\mathrm{NADH}$ and $\mathrm{NAD}^{+}$: spectral and kinetic characterization of intermediates. Biochemistry 33, 12184-12193

30. Keirns, J. J., and Wang, J. H. (1972) Studies on nicotinamide adenine dinucleotide phosphate reductase of spinach chloroplasts J. Biol. Chem. 247, 7374-7382

31. Ballou, D., and Batie, C. J. (1988) Phthalate oxygenase, a Rieske iron-sulfur protein from Pseudomonas cepacia. In Oxidases and Related Redox Systems (King, T. E., Mason, H. S., and Morrison, M., eds) pp. 211-226, Alan R. Liss, Inc. New York

32. Bertrand, P., More, C., and Camensuli, P. (1995) Evidence for a magic magnetic between FMN and the $[2 \mathrm{Fe}-2 \mathrm{~S}]^{+}$center of phthalate dioxygenase reductase of Pseudomonas cepacia. J. Am. Chem. Soc. 117, 1807-1809

33. Ballou, D. P., Gassner, G. T., Wang, L., Batie, C., Gatti, D., Dunham, W. R. and Sands, R. H. (1994) Physical studies on phthalate dioxygenase reductase. In Flavins and Flavoproteins 1993 (Yagi, K., ed) pp. 639-647, K' alter de Gruyter and Co., Berlin

34. Batie, C. J., and Ballou, D. P. (1990) Phthalate dioxygenase. Methods Enzymol. 188, 61-70

35. Gassner, G. T. (1995) Mechanistic studies of the phthalate deoxygenase system, Ph.D. dissertation, The University of Michigan, Ann Arbor

36. Batie, C. J., and Kamin, H. (1986) Association of ferredoxin-NADP ${ }^{+}$reductase with NADP(H): specificity and oxidation reduction properties J. Biol. Chem. 259, 11976-11985

37. Lambeth, D. J., and Kamin, H. (1976) Adrenodoxin Reductase: properties of the complexes of reduced enzyme with NADP ${ }^{+}$and NADPH. J. Biol. Chem. 251, 4299-4306

38. Correll, C. C., Gatti, D. L., and Ludwig, M. L. (1994) Oxidized, reduced, and liganded states of the iron-sulfur flavoprotein, phthalate dioxygenase reductase. In Flavins and Flavoproteins 1994 (Yagi, K., ed) pp. 649-658, Walter de Gruyter and Co., Berlin

39. Marcus, R. A., and Sutin, N. (1985) Electron transfers in biology and chemistry. Biochim. Biophys. Acta 811 , 265-322

40. Moser, C.C., Keske, J. M., Warncke, K., Farid, R.S., and Dutton, L. P. (1992) Nature of biological electron transfer. Nature (London) 355, 796-802

41. Hoffman, B. M., and Ratner, M. A. (1987) Gated electmn transfer: when are observed rates controlled by conformational interconversion? J. Am. Chem. Soc. 109, 6237-6243 